\title{
A INFLUÊNCIA DO INTERVALO DE DESCANSO ENTRE A REPETIÇÃO DE UM TESTE DE RESISTÊNCIA MUSCULAR LOCALIZADA EM DUAS DIFERENTES FERRAMENTAS
}

Jaqueline Santos Silva ${ }^{1}$, Gabriela Carrion Caldeira Ribeiro ${ }^{2}$, Vitoria de Cascaes Zambon ${ }^{2}$, Aryane Flauzino Machado ${ }^{1}$, Jéssica Kirsch Micheletti ${ }^{1}$, Larissa Rodrigues Souto ${ }^{3}$, Gabriela Carvalho ${ }^{2}$, Amanda Paula Balan ${ }^{2}$, Camila Freitas de Souza ${ }^{2}$, Jayme Netto Junior ${ }^{4}$, Carlos Marcelo Pastre ${ }^{4}$

Universidade Estadual Paulista - UNESP, ${ }^{1}$ Programa de Pós-Graduação em Fisioterapia, ${ }^{2}$ Curso de Graduação em Fisioterapia, ${ }^{3}$ PósGraduação Lato Sensu em Fisioterapia Desportiva, ${ }^{4}$ Departamento de Fisioterapia, Laboratório de Fisioterapia Desportiva-LAFIDE, Presidente Prudente, SP. e-mail: jaqueee-santosss@hotmail.com

\section{RESUMO}

O objetivo deste estudo foi verificar a porcentagem de indivíduos recuperados passados cinco minutos após a realização de teste de resistência a fadiga em duas diferentes ferramentas. Amostra foi composta por 13 participantes, submetidos a um teste de resistência muscular localizada em dois dias, com intervalo de sete dias entre eles. O teste foi realizado no tubo elástico e no dinamômetro isocinético, caracterizando um estudo cross over. No tubo elástico $84,6 \%$ dos participantes se recuperaram no intervalo de cinco minutos em ambos os dias de teste. No dinamômetro isocinético o primeiro dia de teste teve $69,2 \%$ dos participantes recuperados. Enquanto que no segunda dia de teste, o percentual de participantes recuperados atingiu valor de $76,9 \%$. Como conclusão, verificou-se que maior percentual de indivíduos se recuperam após realizarem o teste de resistência muscular localizada a fadiga no tubo elástico, porém tais diferenças não foram significantes, que pode ser justificada pelo tamanho da amostra. Por fim, pode-se concluir que cinco minutos seja suficiente para recuperar indivíduos fisicamente ativos.

Palavras-chave: treinamento de resistência, aptidão física, exercício, teste de resistência, intervalo de descanso.

\section{THE REST PERIOD OF INFLUENCE BETWEEN THE REPEAT OF A MUSCULAR ENDURANCE TEST LOCATED IN TWO DIFFERENT TOOLS}

\begin{abstract}
The objective of this study was to verify the percentage of individuals recovered after five minutes, after performing the same test on two different tools at different times. Participants $(n=13)$ will perform the muscular endurance test localized fatigue, on two occasions, with an interval of seven days between them. Everyone will accomplish so much in the elastic tube, as in the isokinetic dynamometer. In the elastic tube $84.6 \%$ of participants recovered at the fiveminute interval in both test days. In the isokinetic dynamometer the first test day had $69.2 \%$ of the participants recovered. While in the second test day, the percentage of recovered participants reached value $76.9 \%$. Thus, as the conclusion, it was found that the highest percentage of individuals recover after performing the muscular endurance test localized fatigue in the elastic tube, but these differences are not very significant, and can be justified by the sample size. Finally, it can be concluded that five minutes is sufficient to recover physically active subjects.
\end{abstract}

Keywords: resistance training, physical fitness, exercise, test of resistance, rest interval.

\section{INTRODUÇÃO}

O intervalo de recuperação entre um exercício e outro, pode exercer influências com relação ao número de repetições realizadas em cada série. Assim, estudos sugerem que maior intervalo de descanso (de 2 a 5 minutos) entre as séries seja responsável por maior número de repetições quando comparados a intervalos mais curtos (de 1 minuto) ${ }^{1,2,3}$. Além disso, extensão do intervalo de recuperação pode proporcionar melhor recuperação, por meio da maior remoção de catabólitos, que minimiza o estresse e a dor muscular tardia 4 .
De acordo com levantamento realizado na literatura, foi possível verificar que a maior parte dos testes realizados adota intervalo curto aqueles que utilizam 1 minuto e intervalo longo o tempo que varia entre 2 e 3 minutos. Ademais, intervalos longos são responsáveis por maior número de repetições ${ }^{1,2,3}$. Neste cenário, sendo o teste analisado de resistência, é evidente a importância de intervalo maior, para execução de mais repetições, durante maior intervalo de tempo.

Segundo Fleck e Kraemer, a manipulação cuidadosa dos períodos de descanso é essencial 
para evitar que se coloque uma tensão inadequada e desnecessária no individuo durante o treinamento ${ }^{5}$. Sabendo-se tratar de um teste desenvolvido recentemente e com informações científicas em processo de elaboração quanto aos reais benefícios, tal estudo foi formulado a partir de dados de um projeto piloto, a fim de levantar informações na literatura acerca do tema e garantir que o intervalo de recuperação proposto seja seguro. Para tanto, será analisado o intervalo de cinco minutos, utilizado a partir de levantamento prévio de estudos da mesma natureza, onde foi evidenciada a manipulação de tal intervalo entre aplicação de um mesmo teste ${ }^{6}$.

$O$ teste desenvolvido vem sendo reproduzido e analisado em duas ferramentas diferentes, o tubo elástico e o dinamômetro isocinético. Sendo assim, ressalta-se a importância de verificar se o intervalo de recuperação é o mesmo para ambas as ferramentas, pois ainda que, o teste seja executado de maneira similar, a peculiaridade de cada ferramenta pode interferir no tempo de recuperação. Neste cenário, este estudo tem como objetivo analisar a influência do intervalo de descanso entre a execução do mesmo teste de resistência muscular localizada em duas diferentes ferramentas.

\section{MATERIAL E MÉTODOS}

A casuística do estudo foi composta por 13 indivíduos do sexo masculino com idade entre
18 e 30 anos, estudantes universitários e aparentemente saudáveis com base nas informações auto-relatadas ${ }^{7}$. Foram considerados como critérios de exclusão características como: ser etilista, consumir drogas, fumo ou medicamentos anti-inflamatórios de forma crônica, apresentar anemia, processo inflamatório, diabetes, doença cardiovascular, problemas no fígado e episódio de lesão músculo-tendínea ou osteoarticular nos membros inferiores e/ou coluna nos últimos seis meses. Nenhum participante foi excluído.

Os participantes foram informados sobre os procedimentos e objetivos do estudo e, após concordarem, assinaram um termo de consentimento livre e esclarecido assegurando sua privacidade. $O$ projeto foi submetido ao comitê de ética da Faculdade de Ciências e Tecnologia (FCT/UNESP) sob $\mathrm{n}^{\circ}$ de CAAE: 46617815.1.0000.5402.

Delineamento do estudo

A coleta de dados foi realizada num centro de atendimento em Fisioterapia e Reabilitação da FCT/UNESP, respeitando o horário das $17 \mathrm{~h} 00$ às $20 \mathrm{~h} 00$.

O estudo foi cross-over, ou seja, todos os participantes em ocasiões diferentes realizaram o teste nas duas ferramentas analisadas. 0 desenho do estudo com as etapas e intervalos mensurados são demonstrados na Figura 1.

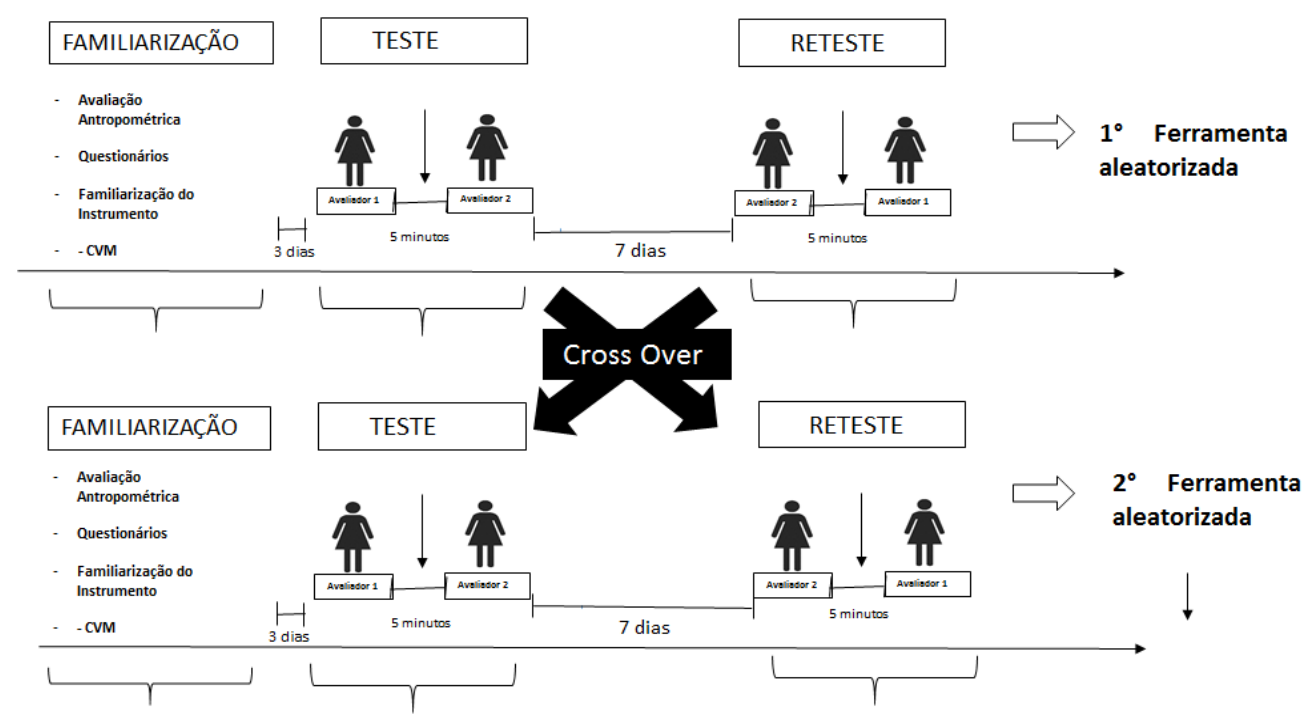

Figura 1. Desenho de estudo. 
Em todas as sessões de teste, foi inserido, após a aplicação do primeiro avaliador, cinco minutos de descanso e logo após, aplicação de uma escala de Percepção de esforço de Likert antes que o segundo avaliador inicie o teste.

\section{Procedimentos}

\section{Percepção de recuperação}

A percepção de recuperação do membro inferior dominante foi avaliada por meio da Escala de Percepção de Esforço de Likert, que consiste em uma escala ordinal com valores entre 1 e 10 pontos, sendo 1 correspondente a nenhuma recuperação e 10 a totalmente recuperado. Para aplicação da escala o participante respondeu a seguinte pergunta: “De 1 a 10 pontos, como você classifica a sua percepção de recuperação em seus membros inferiores para realizar 0 mesmo teste novamente, sendo 1 correspondente a nenhuma recuperação e 10 a totalmente recuperado?". Todas as respostas foram anotadas em fichas individualizadas de acordo com o momento de cada coleta (teste e reteste). Tal dinâmica de coletas já foi realizada no estudo de Buchheit et al. ${ }^{8}$

Teste de Resistência Muscular Localizada à Fadiga Trata-se de um teste desenvolvido pelo Laboratório de Fisioterapia Desportiva (LAFIDE) da FCT/UNESP - Presidente Prudente, utilizado para prescrição do treinamento para ganho de resistência muscular localizada, que determina o volume e a intensidade a serem seguidos progressivamente.

O participante foi instruído a realizar o máximo de repetições que consiga, livre de sinais e sintomas, com elevada frequência e mantendose no mesmo ritmo empreendido desde o início do teste. Para as repetições serem válidas, foi necessário realizar o movimento na amplitude completa e sem qualquer tipo de compensação, respeitando o ritmo constante. Quando tais requisitos deixaram de serem cumpridos o teste foi interrompido e refeito novamente após cinco minutos, caso o tempo atingido não esteja no intervalo esperado. $O$ terapeuta julgou $O$ cumprimento dos requisitos descritos.
Preconiza-se que o teste seja realizado em um intervalo de tempo entre 40 e 75 segundos, pois durante este período o gasto energético é predominantemente anaeróbico e, portanto, este é o tempo para alcance da fadiga ${ }^{9}$. Caso o participante conseguisse realizar o teste por mais tempo, as reservas energéticas utilizadas seriam outras, indicando que a resistência imposta estava muito leve. Por outro lado, se a fadiga ocorrer antes dos 40 segundos era um indício de que a resistência estava além de sua capacidade funcional. Nestes casos, quando o teste não é realizado no intervalo de tempo esperado, uma nova tentativa foi realizada, após cinco minutos de intervalo.

Foi realizado imediatamente ao início dos testes um aquecimento constituído por dez repetições de flexo-extensão do membro dominante, sem carga. Este teste foi desenvolvido para posterior aplicação de um protocolo de treinamento para ganho de resistência muscular localizada. 0 mesmo está sendo desenvolvido e testado em coleta de dados, e posteriormente terá seus dados divulgados.

\section{RESULTADOS}

Os valores da Escala de Percepção de Esforço de Likert cinco minutos após realizado o teste de resistência muscular localizada à fadiga são apresentados na tabela 1 e 2 sendo que, a tabela 1 representa valores do tubo elástico e a tabela 2, representa valores do dinamômetro isocinético.

A Tabela 1 demonstra os valores de likert para o tubo elástico. Em ambos os testes, a porcentagem de participantes que se recuperaram no intervalo de 5 minutos foram a mesma, correspondendo $84,6 \%$ dos participantes.

A Tabela 2 demonstra os valores de recuperação no dinamômetro isocinético. No primeiro dia de teste, este valor foi de $69,2 \%$. Enquanto que no segundo dia de teste, o percentual de participantes recuperados atingiu valor de $76,9 \%$. 
Tabela 1. Valores de Escala de Percepção de Esforço de Likert após realização do teste no tubo elástico.

\begin{tabular}{|c|c|c|}
\hline Participante & Primeiro dia de teste & Segundo dia de teste \\
\hline 1 & 8 & 4 \\
\hline 2 & 7 & 9 \\
\hline 3 & 9 & 9 \\
\hline 4 & 8 & 7 \\
\hline 5 & 9 & 7 \\
\hline 6 & 10 & 10 \\
\hline 7 & 8 & 9 \\
\hline 8 & 9 & 10 \\
\hline 9 & 8 & 6 \\
\hline 10 & 5 & 8 \\
\hline 11 & 7 & 8 \\
\hline 12 & 8 & 7 \\
\hline 13 & 6 & \\
\hline
\end{tabular}

Tabela 2. Valores de Escala de Percepção de Esforço de Likert após realização do teste no Dinamômetro Isocinético.

\begin{tabular}{|c|c|c|}
\hline Participante & Primeiro dia de teste & Segundo dia de teste \\
\hline 1 & 4 & 5 \\
\hline 2 & 3 & 6 \\
\hline 3 & 5 & 4 \\
\hline 4 & 5 & 7 \\
\hline 5 & 7 & 7 \\
\hline 6 & 10 & 10 \\
\hline 7 & 8 & 8 \\
\hline 8 & 7 & 7 \\
\hline 9 & 8 & 10 \\
\hline 10 & 8 & 7 \\
\hline 11 & 7 & 8 \\
\hline 12 & 7 & 7 \\
\hline 13 & 7 & 8 \\
\hline
\end{tabular}

\section{DISCUSSÃO}

O presente estudo teve como objetivo principal analisar os níveis de recuperação após um teste de resistência muscular localizada, com intervalo de cinco minutos, realizados em duas ferramentas e comparar os níveis de recuperação entre ambos. Os principais achados demostraram que a partir do intervalo de repouso de cinco minutos, o percentual de indivíduos recuperados é maior no tubo elástico.

Crisafulli et al. ${ }^{4}$, em um estudo com atletas, concluíram que quando utilizado menor intervalo de recuperação, pode ocorrer um aumento no acúmulo de metabólitos que são produzidos durante a contração muscular diminuindo a resistência periférica vascular ${ }^{4}$. Tal mecanismo pode ser responsável por uma performance diminuída durante competições ou ainda ser um fator para predisposição de algum tipo de lesão musculoesquelética, o que remete a importância de manipulação correta quanto a intervalos de recuperação.

Na primeira ferramenta analisada, o tubo elástico, o intervalo aplicado foi suficiente para recuperação de $84,6 \%$ dos indivíduos. Tal resultado corrobora com achados no estudo de Paula et al. ${ }^{10}$ que analisou diferentes marcas de tubos elásticos, concluindo que os tubos perdem sua capacidade de responder com os mesmos valores força após clivagem, oferecendo menor resistência após certo tempo, portanto, um menor grau de fadiga aos indivíduos. Já na segunda ferramenta, dinamômetro isocinético, a resistência varia de acordo com a força aplicada pelo participante. Os estudos de Ernesto ${ }^{11}$ mostraram que o intervalo de recuperação 
exerce importante influencia sobre o desempenho muscular isocinético em idosos principalmente a partir da $3^{\circ}$ serie. Parcell et al. ${ }^{11}$ analisaram diferentes intervalos de recuperação $(15,60,180,300 \mathrm{seg})$ em jovens, demonstrando que o IR de 60 segundos ser o mais eficaz no que se refere a uma recuperação completa na avaliação do PT (pico de torque) durante contrações isocinéticas.

As evidências a partir dos achados apontam para uma conclusão de que após execução de um mesmo teste, a maioria dos indivíduos se recuperou após o intervalo de cinco minutos. Porém, ainda que o percentual de sujeitos recuperados seja maior no tubo elástico, a diferença entre estas duas ferramentas é baixa. Além disso, após levantamento realizado na literatura sobre o tema e partindo do princípio que o estudo foi realizado apenas com amostra composta por participantes fisicamente ativos, acredita-se que o intervalo de cinco minutos seja suficiente para recuperação em tal perfil de amostra uma vez que, o teste aplicado não oferece grandes níveis de exaustão, além de ser baseado em parâmetros energéticos com princípios de evitar níveis alterados de fadiga.

\section{CONCLUSÃO}

Verificou-se que maior percentual de indivíduos se recuperam após realizarem o teste de resistência muscular localizada a fadiga no tubo elástico, porém tais diferenças não foram significantes, e pode ser justificada pelo tamanho da amostra. Por fim, pode-se concluir que cinco minutos seja suficiente para recuperar indivíduos fisicamente ativos.

\section{CONFLITO DE INTERESSE}

Os autores declaram não haver qualquer potencial de conflito de interesse que possa interferir na imparcialidade deste trabalho científico.

\section{REFERÊNCIAS}

1. Alves RM, Sobreira R, Castro JS, Zovico PV, Oliveira W, Curty VM. Efeito do exercício com 1 e 3 minutos de intervalo de descanso entre as séries na atividade sérica das transaminases. Acta Biom Brasiliensia. 2011; 2(2):1-10.

2. Pereira MIR, Gomes PSC, Bhambhanil Y. Número máximo de repetições em exercícios isotônicos: influência da carga, velocidade e intervalo de recuperação entre séries. Rev Bras Med Esporte. 2007;13(5):287-91.

DOI:

http://dx.doi.org/10.1590/S1517-

86922007000500002

3. Simão R, Polito M, Monteiro W. Efeito de diferentes intervalos de recuperação em um programa de treinamento de força para indivíduos treinados. Rev Bras Med Esporte. 2008;14(4):353-6. DOI: http://dx.doi.org/10.1590/S1517-

\section{6}

4. Polito DM. Pressão arterial, frequência cardíaca e duplo-produto em séries sucessivas do exercício de força com diferentes intervalos de recuperação. Rev Portug Ciên Desporto. 2004;4(3):7-15.

5. Simão R, Monteiro $W$, Jacometo $A$, Tesseroli $C$, Teixeira G. A influência de três diferentes intervalos de recuperação entre séries com cargas para 10 repetições máximas. Rev Bras Ciên Mov. 2006;14(3): 37-44.

6. Alves HB, Simão R, Dias MR. Número de repetições e percentual de carga máxima: comparação entre exercício uni e multiarticular. Rev Bras Prescr Fisiol Exerc. 2012;32(6):157-63.

7. Interdonato GC, Greguol M. Qualidade de vida percebida por indivíduos fisicamente ativos e sedentários. Rev Bras Ciên Mov. 2010;18(1):61-7.

8. Buchheit M, Peiffer JJ, Abbiss CR, Laursen PB. Effect of cold water immersion on postexercise parasympathetic reactivation. Am J Physiol Heart Circ Physiol. 2009;296:421-7.

9. Foss MK, Fox, S. Bases Fisiológicas do Exercício e do Esporte. 6.ed. Rio de Janeiro: Editora GuanabaraKoogan; 2000.

10. Paula LV, Santos SS, Araújo CM. Resistência e fadiga de tubos elásticos utilizados em reabilitação e treinamento esportivo. FIEP Bulletin. 2010;80:1-10.

11. Ernesto C, Bottaro M, Silva FM, Sales MPM, Celes RS, Oliveira RJ. Efeitos de diferentes intervalos de recuperação no desempenho muscular isocinético em idosos. Braz J Phys Ther. 2009;13(1):65-72.

Recebido para publicação em 19/08/2015

Revisado em 09/09/2015

Aceito em 18/09/2015 